\title{
Expression of putative stem marker nestin and CD133 in advanced serous ovarian cancer
}

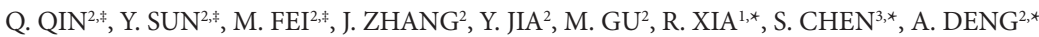 \\ ${ }^{1}$ Department of Transfusion, Huashan Hospital, Fudan University, Shanghai 200040, China; ${ }^{2}$ Department of Laboratory Diagnostic, Changhai \\ Hospital, Second Military Medical University, Shanghai 200433, China; ${ }^{3}$ Changzheng Hospital, Second Military Medical University, Shanghai \\ 200433, China
}

*Correspondence: anmeideng@yahoo.com.cn,rongxia10@163.com,chensunxiao@126.com

${ }^{*}$ Contributed equally to this work.

Received November 7, 2011 / Accepted December 10, 2011

\begin{abstract}
It is hypothesized that "cancer stem cells" are responsible for the resistance to chemotherapy of cancer cells in ovarian cancers. The objective of the studies was to explore if the stem cell biomarkers could be used to predict the tumor chemotherapy-resistance in serous ovarian cancer patients. Expression of two putative stem cell markers CD133 and nestin, and vascular epithelial growth factor (VEGF) and epidermal growth factor receptor (EGFR) were detected in 123 cases of advanced serous ovarian cancer specimens by immunohistochemistry. To estimate intra-tumoral microvessel density (MVD), CD34 immunostaining was also performed. CD133 and nestin were defined to be positive in $35.0 \%$ and $32.5 \%$ of the serous ovarian carcinoma tissues, respectively. It was observed that overexpression of nestin but not CD133 was associated with the cisplatin-based chemotherapy resistance and shorter overall survival of the patients, and nestin was found to be an independent prognostic factor. Moreover, positive nestin expression also correlated to increased expression of EGFR and VEGF, and elevated MVD in tumors. The results of this study suggest that serous ovarian cancers with high expression level of nestin represent an aggressive malignant phenotype associated with poor prognosis, and treatment targeted the nestin positive cancer cells might be a promising therapeutic strategy for this subgroups.
\end{abstract}

Key words: ovarian carcinoma, nestin, CD133, chemotherapy, resistance

Ovarian carcinoma is one of the most common causes of cancer deaths in gynaecological malignancies worldwide. Besides surgical procedures, adjuvant taxol or cisplatinbased chemotherapy is often administered, especially in the advanced stages of ovarian cancer. However, resistance to chemotherapy is of major concern, leading to a high relapse rate (approximately $60-75 \%$ ) and a poor prognosis [1]. Until now, there is still a lack of biomarkers that can accurately predict tumor response to chemotherapy in ovarian cancer.

Currently, increasing evidence suggests that a minor population of "cancer stem cells" capable of self-renewal and unlimited proliferation, is responsible for the resistance to therapy of cancer cells [2]. Several cancer stem markers have been identified to be possible chemoresistance markers in a few solid tumors $[3,4]$. Based on above findings, we hypothesized that cancer stem cell markers could also be used to predict chemotherapy response in ovarian cancer.

The predictive value of stem markers for the chemotherapy resistance in ovarian cancer has not been reported before. Therefore, in this present study, we investigated the expression of two putative stem biomarkers CD133 and nestin in a cohort of advanced serous ovarian cancer tissues by immunohistochemistry, and their chinicopathological and prognostic significance was also evaluated.

\section{Patients and methods}

Patients and specimens. A total of 123 formalin-fixed and paraffin-embedded tumor tissues were collected from advanced (Stage III and IV) serous ovarian carcinoma patients operated at the Huashan Hospital, Fudan University(Shanghai, 
China), Changhai Hospital, Second Military Medical University(Shanghai, China), and Changzheng Hospital, Second Military Medical University, (Shanghai, China) with informed consent. This present study was approved by the local ethics committees. Histopathology, grade and disease stage of ovarian cancer patients were determined according to WHO criteria, Gynecologic Oncology Group criteria, and the International Federation of Gynecology and $\mathrm{Ob}$ stetrics (FIGO) system, respectively. All the ovarian cancer patients received cisplatin-based postoperative adjuvant chemotherapy, and response to chemotherapy was assessed according to WHO criteria. Patients with complete response (CR) and partial response (PR) were defined as "response to chemotherapy", while patients with stable disease (SD) or progressive disease (PD) were classified as "no-response to chemotherapy". Overall survival (OS) was calculated from the date of histological diagnosis to the date of death or last follow-up examination.

Immunohistochemistry. Immunohistochemistry was performed to detect the expression of CD133, nestin, vascular epithelial growth factor (VEGF), epidermal growth factor receptor (EGFR) and CD34 in formalin-fixed paraffin-embedded ovarian cancer specimens, as follows. Briefly, $4 \mu \mathrm{m}$ sections were deparaffinized with xylene, then were treated with $0.3 \%$ hydrogen peroxide for $30 \mathrm{~min}$ to inactive endogenous peroxidases. The slides were incubated with normal goat serum for $1 \mathrm{~h}$ to block nonspecific binding, and then were incubated overnight at $4^{\circ} \mathrm{C}$ using anti-CD133 antibody (Abcam, 1:100), anti-nestin antibody (Santa Cruz, 1:50), anti-EGFR antibody (Santa Cruz, 1:100) or anti-CD34 (Dako, 1:100). The specificities of the antibodies have been confirmed in previous studies. Primary antibodies were detected using HRP Envision Systems (Dako, Gene Co. Ltd., Shanghai, China), and then were counterstained with haematoxylin. For the negative controls, the primary antibodies were replaced by mouse or rabbit IgG.

Evaluation of immunohistochemical results. Immunohistochemical results were evaluated according to the following criteria as described previously. Cases were defined as positive for the expression of CD133 and nestin when more than $10 \%$ of tumor cells demonstrated a moderate to strong staining intensity; the others were scored as negative expression. The cut-off value of $10 \%$ was selected for the assumption that $10 \%$ of nestin or CD133 positive cancer cells may be enough to exert the "stem" effect in cancer tissues [5]. The cut-off point distinguishing between positive and negative VEGF expression was $\geq 30 \%$ [6]. Microvessel density (MVD) was determined by CD34-positive microvessel counting at $\times 200$ magnification. A mean value was used to categorize ovarian tumors into low and high MVD subgroups.

Statistical analysis. The difference between groups with and without each stem marker expression was compared with using $\chi 2$ test or Fisher's exact test as appropriate. OS curves were estimated by the Kaplan-Meier methods and compared using the log-rank test. Multivariate analyses of survival were carried out using the Cox regression model. Statistical analyses were performed using SPSS V.13.0 software. P values of less than 0.05 were considered statistically significant.

\section{Results}

Both CD133 and nestin stained the cytoplasm and/or membrane of tumor cells with a variety of staining patterns, including focal, scattered and diffuse staining (figure 1). According to the criteria for stem cell biomarker immunostaining established, positive CD133 and nestin expression were defined in $35.0 \%(43 / 123)$ and $32.5 \%(40 / 123)$, of the serous ovarian tumors, respectively. Positive VEGF and EGFR expressions were defined in $65.9 \%$ and $46.3 \%$, respectively. 56 patients were categorized as high MVD and 67 as low MVD based on a mean MVD value of 75.8. Representative images of immunostaining of ovarian cancer tissues were shown in figure 1.

No significant association was found between CD133 and nestin expression with all the clinicopathological characteristics, except that nestin overexpression correlated with response to chemotherapy (table 1). Nestin-positive cases had a higher incidences of non-response to chemotherapy than nestin-negative subgroup ( $55.0 \%$ vs. $20.1 \%, \mathrm{P}=0.001)$. The correlation of the CD133 and nestin expression with tumor angiogenesis and EGFR expression in serous ovarian carcinomas was further evaluated. Tumors in the nestin-positive group expressed higher level of VEGF and had a significantly higher MVD than those in the nestin-negative group $(\mathrm{P}=0.001$, $\mathrm{P}=0.014$, respectively). EGFR overexpression was more frequently observed in the nestin-positive tumors (77.5\%) than the nestin-negative subgroup $(31.3 \%, \mathrm{P}=0.001)$. There was no significant difference in tumor angiogenesis and EGFR overexpression between the CD133-positive and -negative groups. EGFR positive subgroup had a trend toward high level of VEGF expression and increased MVD, however, statistical significance was not reached.

Overall survival curves were estimated according to the expression status of stem markers CD133 and nestin, and then compared using the log-rank test. The nestin-positive patients had a significantly shorter overall survival times than the negative subgroup, meanwhile CD133 expression had no impact on the survival in patients with serous ovarian carcinomas (table 2, figure 2). Cox proportional hazards analysis demonstrated that nestin expression was retained as an independent prognostic factor of shorter overall survivals for the ovarian carcinoma patients (table 2).

\section{Discussion}

Ovarian carcinoma is highly heterogeneous in tumor progression and response to treatment. Most commonly used subgroup classification systems are based on the clinical characteristics (such as histological type, FIGO stage); however, such classification criteria lack prognostic significance in 


\section{Positive}

CD133

Nestin

EGFR

VEGF
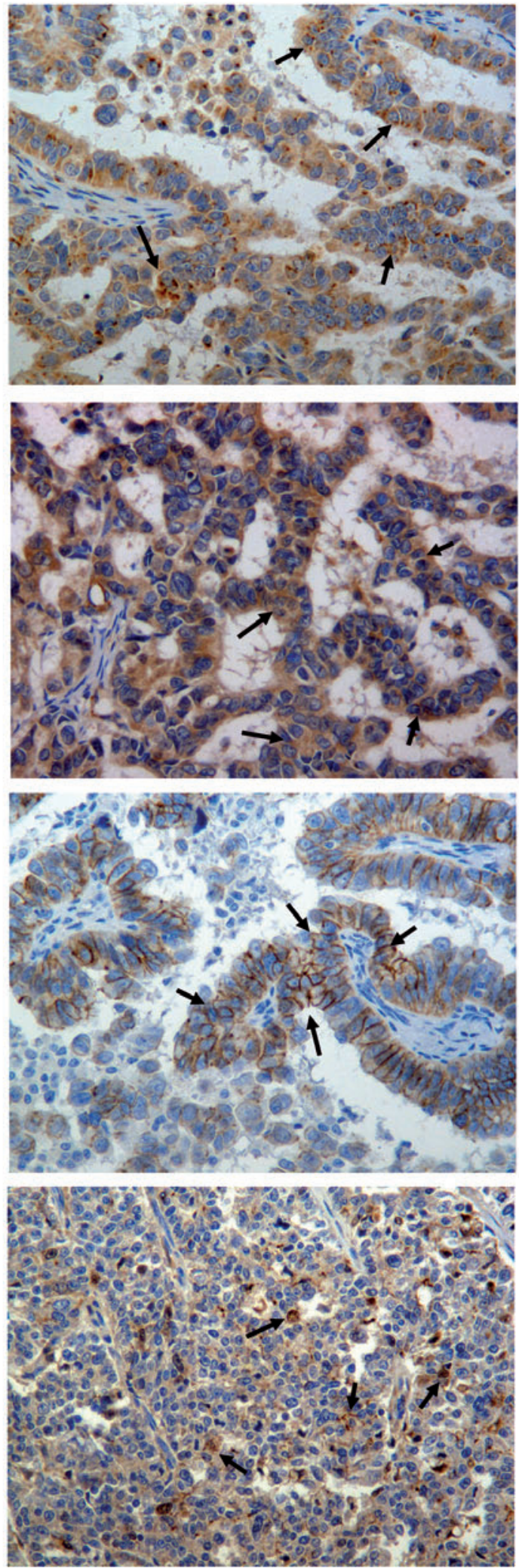

\section{Negative}
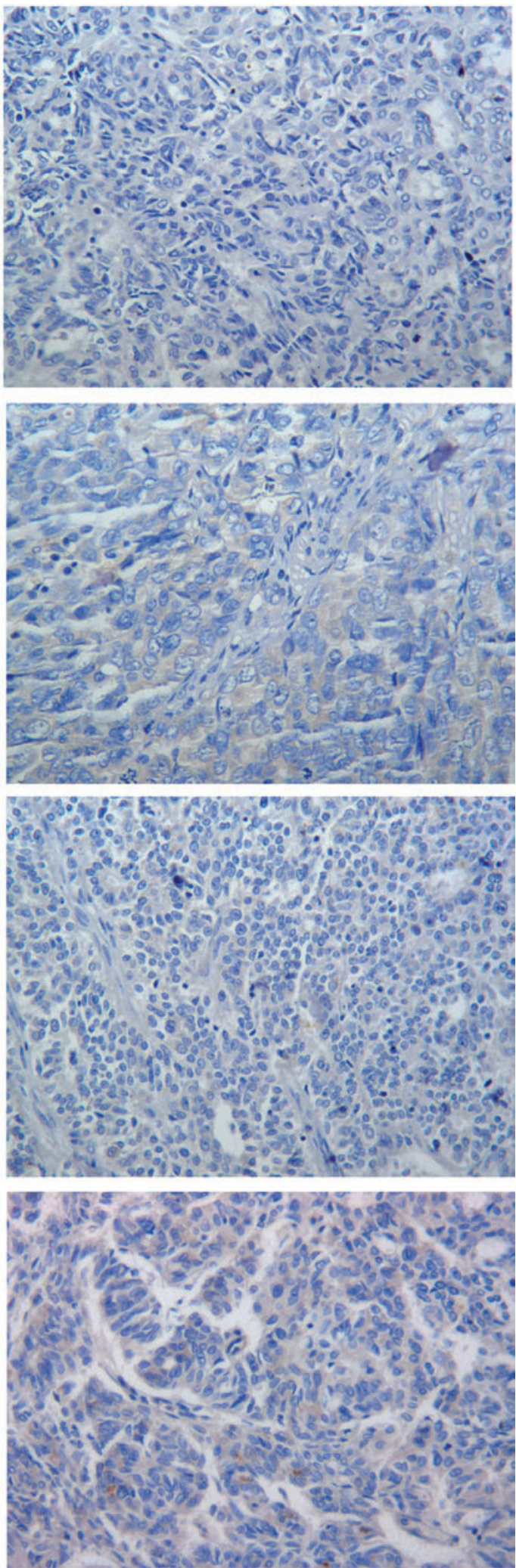

Figure 1. Arrows indicated the immunohistochemical staining of CD133, nestin, EGFR and VEGF protein in serous ovarian cancer tissues $(\times 200)$. 
Table 1. Correlation between the expression of CD133 and nestin with clinicopathological charaterisitics in 123 serous ovarian carcinomas.

\begin{tabular}{|c|c|c|c|c|c|c|c|}
\hline & \multirow[b]{2}{*}{ Total } & \multicolumn{2}{|c|}{ CD133 } & \multirow[b]{2}{*}{$P$ value } & \multicolumn{2}{|c|}{ Nestin } & \multirow[b]{2}{*}{ P value } \\
\hline & & positive & negative & & positive & negative & \\
\hline \multicolumn{8}{|l|}{ Age (yrs) } \\
\hline$<65$ & 87 & 32 & 55 & 0.510 & 15 & 21 & 0.164 \\
\hline$\geq 65$ & 36 & 11 & 25 & & 25 & 62 & \\
\hline \multicolumn{8}{|l|}{ Grade } \\
\hline G1-2 & 93 & 35 & 58 & 0.273 & 33 & 60 & 0.217 \\
\hline G3 & 30 & 8 & 22 & & 7 & 23 & \\
\hline \multicolumn{8}{|l|}{ Stage } \\
\hline III & 92 & 32 & 60 & 0.944 & 31 & 61 & 0.632 \\
\hline IV & 31 & 11 & 20 & & 9 & 22 & \\
\hline \multicolumn{8}{|c|}{ Response to treatment } \\
\hline Yes & 84 & 27 & 57 & 0.336 & 18 & 66 & 0.001 \\
\hline No & 39 & 16 & 23 & & 22 & 17 & \\
\hline \multicolumn{8}{|c|}{ EGFR expression } \\
\hline Positive & 57 & 24 & 33 & 0.123 & 31 & 26 & 0.001 \\
\hline Negative & 66 & 19 & 47 & & 9 & 57 & \\
\hline \multicolumn{8}{|c|}{ VEGF expression } \\
\hline Positive & 83 & 32 & 51 & 0.108 & 33 & 50 & 0.014 \\
\hline Negative & 40 & 11 & 29 & & 7 & 33 & \\
\hline \multicolumn{8}{|l|}{ MVD } \\
\hline High & 56 & 22 & 34 & 0.789 & 26 & 30 & 0.003 \\
\hline Low & 67 & 21 & 46 & & 14 & 53 & \\
\hline
\end{tabular}

Table 2. Univariate and multivariate analysis of overall survival with regard to clinicopathological characteristics and stem cell biomarkers

\begin{tabular}{|c|c|c|c|c|}
\hline \multirow{2}{*}{ Characteristics } & \multicolumn{2}{|c|}{ Univariate analysis } & \multicolumn{2}{|c|}{ Multivariate analysis } \\
\hline & HR (95\% CI) & P-value & HR (95\% CI) & P-value \\
\hline Age (<65 vs. $\geq 65)$ & $1.312(0.765-2.248)$ & 0.324 & $1.278(0.722-2.264)$ & 0.400 \\
\hline Grade (G1-2 vs. G3) & $1.163(0.671-2.019)$ & 0.590 & $0.911(0.504-1.645)$ & 0.757 \\
\hline Stage (III vs. IV) & $1.340(0.750-2.394)$ & 0.323 & $1.351(0.713-2.561)$ & 0.356 \\
\hline Response to treatment (yes vs no) & $0.395(0.229-0.682)$ & 0.001 & $0.619(0.335-1.146)$ & 0.127 \\
\hline EGFR (positive vs.negative) & $2.000(1.231-3.250)$ & 0.005 & $1.143(0.630-2.075)$ & 0.659 \\
\hline VEGF (positive vs. negative) & $1.703(1.022-2.837)$ & 0.041 & $1.342(0.782-2.303)$ & 0.285 \\
\hline MVD (high vs. low) & $2.329(1.435-3.780)$ & 0.001 & $1.993(1.195-3.323)$ & 0.008 \\
\hline CD133 (positive vs. negative) & $1.419(0.857-2.348)$ & 0.174 & $1.433(0.849-2.419)$ & 0.178 \\
\hline Nestin (positive vs. negative) & $3.423(2.052-5.710)$ & 0.001 & $2.501(1.290-4.846)$ & 0.007 \\
\hline
\end{tabular}

the prediction of outcome and therapeutic effects. Recently, several novel molecular classification systems based on tumor biomarkers such as ER, PR, Her2, p53 have been developed [7-8]; however, their prognostic significance is not consistent in different reports.

Cancer stem cells have been supposed to play a key role in the regulation of cancer cells in malignant progression and chemoresistance. Under stem cell-selective conditions, Zhang et al. [9] identified a subpopulation of CD44+CD117+ ovarian cancer-initiating cells (OCICs) with expressed "stemness' properties, demonstrated increased chemoresistance to cisplatin or paclitaxel, and up-regulation of stem cell markers including Bmi-1, Notch-1, Nanog, nestin, ABCG2, and Oct-4. Similarity, under drug selection using cisplatin and paclitaxel, $\mathrm{Ma}$ et al. [10] isolated a subgroup of cancer cells displaying remarkable stem cell properties from human ovarian cancer cell line SKOV3. This subgroup of SKOV3 cells highly expressed a panel of stem cell biomarkers, such as Nanog, Oct4, sox2, 

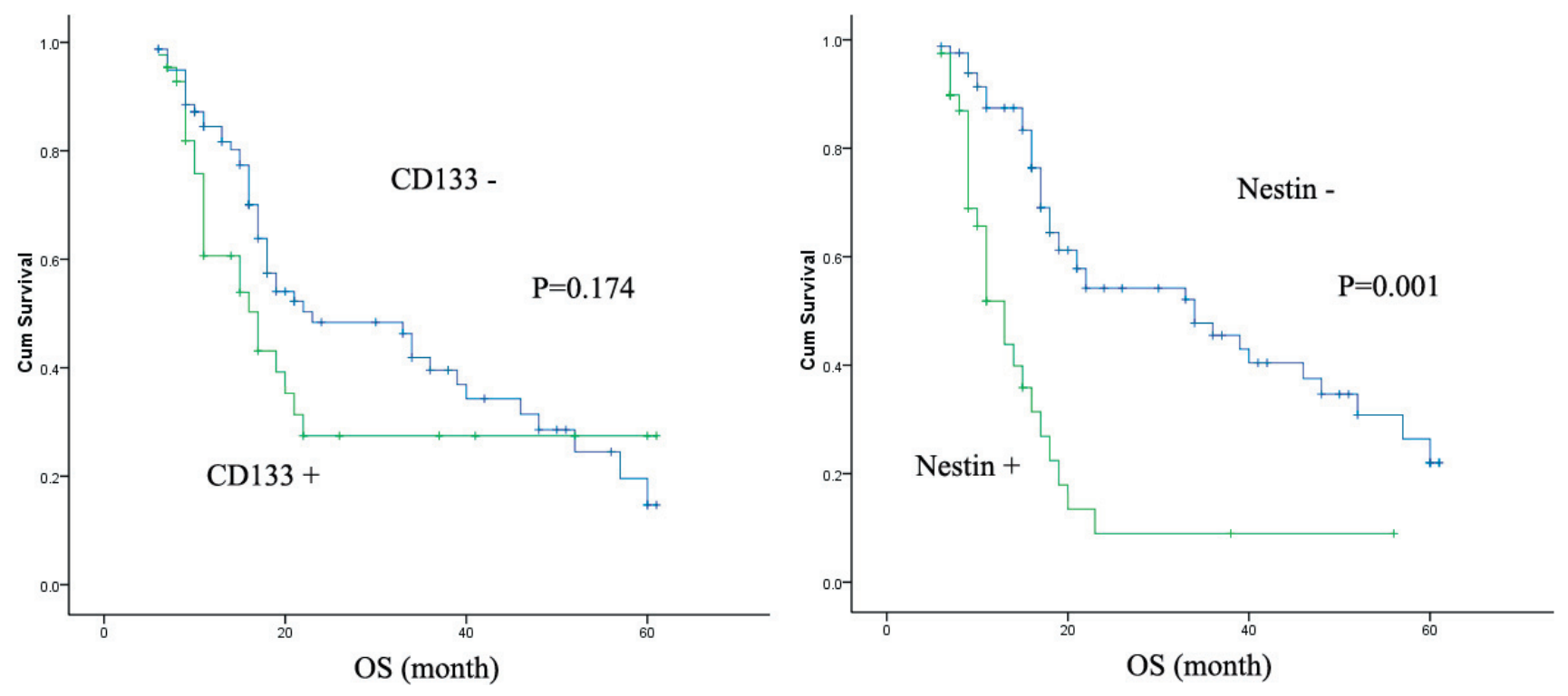

Figure 2. Kaplan-Meier survival curves for overall survival (OS) of patients with serous ovarian cancers according to the expression level of CD133 and nestin.

nestin, ABCG2, CD133, and CD117. Therefore, these findings indicated that cancer stem cell markers could provide a rational source for developing novel phonotype classification criteria for ovarian cancer.

Several previous studies have investigated the clinicopathological significance of stem cell biomarkers in ovarian cancers. For example, Ferrandina et al. [11] found that the CD133 expression seems not to provide additional prognostic information in ovarian cancer patients, however, the diffuse cytoplasmic CD133 pattern might correlate with worse prognosis. Zhang et al. [12] identified that Oct4 was associated with more advanced stage and higher grade, and predicts poor prognosis in serous ovarian carcinoma, but not in mucinous carcinoma.

However, the correlation between stem cell markers and the chemotherapy response in ovarian cancer has not been fully evaluated. In this study, we investigated the expression of two putative stem biomarkers CD133 and nestin in serous ovarian cancer to evaluate if these biomarkers could be used to predict chemotherapy response in clinical practice. Our results showed that no association was observed between CD133 expression and chemotherapy response. Furthermore, CD133 was found to be unrelated to the survival of serous ovarian carcinomas, which is consistent with the previous study [11]. Different from CD133, we identified that nestin expression was significantly associated with chemoresistance in serous ovarian cancers, and also have independent prognostic value in the multivariate analysis. Our results suggest that nestin is capable of identifying a subgroup with poor prognosis and cisplatin-based chemoresistance, which deserves much more intensive therapies.
Cancer stem cells have been linked to EGFR activation in many cancers [13]. Several previous reports have also indicated that cell subpopulations with stem cell properties may contribute to tumor angiogenesis through an induction of VEGF expression or other angiogenic factors [14]. In this study, we found that positive nestin expression was associated with increased EGFR expression and tumor angiogenesis level in serous ovarian cancer tissues. Our findings suggest that a fraction of nestin positive cells might contribute to the malignant development of serous ovarian cancers through EFGR activation and angiogenesis mechanisms.

In conclusion, in this study, we first indicated that the stem cell marker nestin could be used as a novel biomarker to predict cisplatin-based chemotherapy resistance and prognosis for serous ovarian cancer patients. Considering the link between nestin with resistance to cisplatin-based chemotherapies and poor prognosis, we proposed that in the future, treatment targeted the nestin positive fraction in tumors may comprise a promising anti-cancer strategy for patients with serous ovarian carcinomas.

Acknowledgements: This research was supported by grants from National Science Foundation of China (30972730) and Shanghai Municipal Commission for Science and Technology (09JC1405400).

\section{References}

[1] MATSUO K, LIN YG, ROMAN LD, SOOD AK. Overcoming platinum resistance in ovarian carcinoma. Expert Opin Investig Drugs 2010; 19: 1339-54. http://dx.doi.org/10.1517/ $\underline{13543784.2010 .515585}$ 
[2] Alvero AB, CHEN R, FU HH, MONTAGNA M, SCHWARTZ PE, et al. Molecular phenotyping of human ovarian cancer stem cells unravels the mechanisms for repair and chemoresistance. Cell Cycle 2009; 8: 158-66. http://dx.doi. org/10.4161/cc.8.1.7533

[3] HONG SP, WEN J, BANG S, PARK S, SONG SY. CD44positive cells are responsible for gemcitabine resistance in pancreatic cancer cells. Int J Cancer 2009; 125: 2323-31.

[4] TAKAHASHI S, KAMIYAMA T, TOMARU U, ISHIZU A, SHIDA T, et al. Frequency and pattern of expression of the stem cell marker CD133 have strong prognostic effect on the surgical outcome of colorectal cancer patients. Oncol Rep 2010; 24: 1201-12. http://dx.doi.org/10.3892/or 00000973

[5] PIRAS F, PERRA MT, MURTAS D, MINERBA L, FLORIS C, et al. The stem cell marker nestin predicts poor prognosis in human melanoma. Oncol Rep. 2010; 23: 17-24.

[6] LEE TK, POON RT, YUEN AP, LING MT, WANG XH, et al. Regulation of angiogenesis by Id-1 through hypoxia-inducible factor-1alpha-mediated vascular endothelial growth factor upregulation in hepatocellular carcinoma. Clin Cancer Res 2006; 12: 6910-9. http://dx.doi.org/10.1158/1078-0432.CCR-06-0489

[7] GARCIA-VELASCO A, MENDIOLA C, SANCHEZ-MUNOZ A, BALLESTIN C, COLOMER R, et al. Prognostic value of hormonal receptors, p53, ki67 and HER2/neu expression in epithelial ovarian carcinoma. Clin Transl Oncol 2008; 10: 367-71. http://dx.doi.org/10.1007/s12094-008-0213-x

[8] NAKAYAMA K, TAKEBAYASHI Y, NAKAYAMA S, HATA K, FUJIWAKI R, et al. Prognostic value of overexpression of p53 in human ovarian carcinoma patients receiving cisplatin. Cancer Lett 2003; 192: 227-35. http://dx.doi.org/10.1016/ S0304-3835(02)00686-9

[9] ZHANG S, BALCH C, CHAN MW, LAI HC, MATEI D, et al. Identification and characterization of ovarian cancer-initiating cells from primary human tumors. Cancer Res 2008; 68: 431120. http://dx.doi.org/10.1158/0008-5472.CAN-08-0364

[10] MA L, LAI D, LIU T, CHENG W, GUO L. Cancer stemlike cells can be isolated with drug selection in human ovarian cancer cell line SKOV3. Acta Biochim Biophys Sin (Shanghai) 2010; 42: 593-602. http://dx.doi.org/10.1093/ abbs/gmq067

[11] FERRANDINA G, MARTINELLI E, PETRILLO M, PRISCO MG, ZANNONI G, et al. CD133 antigen expression in ovarian cancer. BMC Cancer 2009 7; 9: 221.

[12] ZHANG J, LI YL, ZHOU CY, HU YT, CHEN HZ. Expression of octamer-4 in serous and mucinous ovarian carcinoma. J Clin Pathol 2010; 63: 879-83. http://dx.doi.org/10.1136/ jcp.2009.073593

[13] MARQUARDT JU, RAGGI C, ANDERSEN JB, SEO D, AVITAL I, et al. Human hepatic cancer stem cells are characterized by common stemness traits and diverse oncogenic pathways. Hepatology. 2011 May 26. http://dx.doi. org/10.1002/hep. 24454

[14] ISHIWATA T, MATSUDA Y, NAITO Z. Nestin in gastrointestinal and other cancers: effects on cells and tumor angiogenesis. World J Gastroenterol. 2011; 17: 409-18. http:// dx.doi.org/10.3748/wjg.v17.i4.409 